\title{
Enhanced Resolution for Aquarius Salinity Retrieval near Land-Water Boundaries
}

\author{
Cuneyt Utku \\ USRA/GESTAR \\ Goddard Space Flight Center \\ Greenbelt, MD 20771
}

\author{
David M. Le Vine \\ NASA Goddard Space Flight Center \\ Greenbelt, MD 20771
}

\begin{abstract}
A numerical reconstruction of the brightness temperature is examined as a potential way to improve the retrieval of salinity from Aquarius measurements closer to landwater boundaries. A test case using simulated ocean-land scenes suggest promise for the technique.
\end{abstract}

Keywords-Microwave Remote Sensing; L-Band; Sea Surface Salinity

\section{INTRODUCTION}

The goal of the Aquarius [1] is to map the surface salinity field of the global oceans with a spatial resolution of $150 \mathrm{~km}$ and a monthly RMS accuracy of 0.2 psu [2]. Because of the accuracy requirements for the salinity retrieval and the large footprint of the Aquarius radiometers, the retrieval of salinity is restricted to the open ocean several hundred kilometers from land. At closer distances, land in the antenna side lobes, biases the measurements and causes an underestimation of the salinity field. In principle, it is possible to correct for this "contamination" by land if the antenna pattern and emission from land are well known. However, this is generally not the case. In this study, an alternative approach for retrieving salinity close to land is developed using a regularized reconstruction of the brightness temperature.

\section{AQUARIUS SALINIY RETRIEVAL}

Aquarius radiometers report measurements of the microwave emission from Earth every 1.44 seconds. The basic observation geometry is shown in Fig. 1. After making corrections for interfering sources of radiation, such as the galactic background, the Sun and the atmosphere [3], the antenna temperature at a point along the boresight can be written in the form [4]:

$$
\boldsymbol{T}_{A}\left(\boldsymbol{r}_{i}\right)=\int_{\Omega_{F O V_{i}}} \mathbf{G}\left(\omega, \boldsymbol{b}_{o}\right) \boldsymbol{T}_{B}(\omega) d \omega
$$

where $\boldsymbol{T}_{A}=\left[T_{A H}, T_{A V}\right]^{T}, \boldsymbol{T}_{B}=\left[T_{H}, T_{V}\right]^{T}$ and the antenna pattern matrix pointing in the boresight direction $\boldsymbol{b}_{O}$, is $\mathbf{G}\left(\omega, \boldsymbol{b}_{o}\right)$. Here $\Omega_{F O V_{i}}$ is the solid angle subtended by the visible disk (FOV) corresponding to the $i$ th measurement.

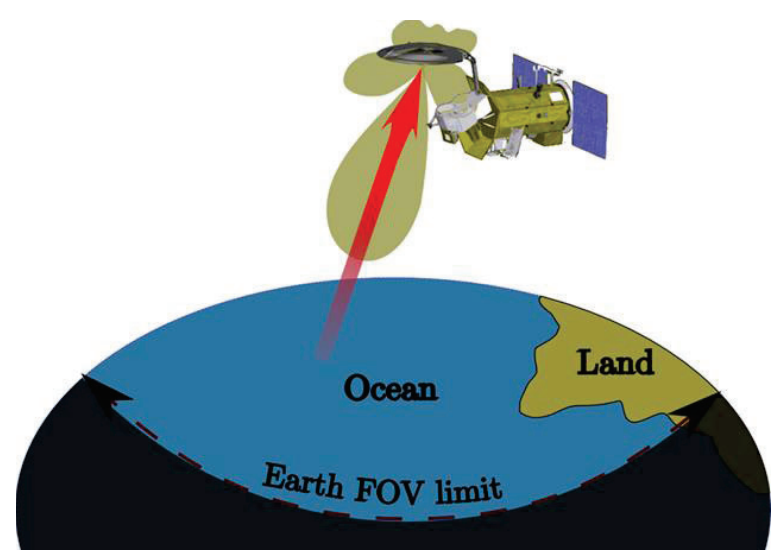

Figure 1: Earth-view geometry

Figure 2 illustrates the problem with land in the field-ofview of the radiometer. The error due to radiation from land is plotted (ordinate) as a function of the distance of the antenna boresight from the land-water boundary (abscissa). In this example, the outer-most Aquarius radiometer beam is used (local incidence angle of 45.6 degrees). An error of $0.1 \mathrm{~K}$ corresponds roughly to the goal of $0.2 \mathrm{psu}$. Hence, the acceptable error is reached at about $450 \mathrm{~km}$ from the landwater boundary which is about 4.5 footprints $(3 \mathrm{~dB})$ for the outer beam.

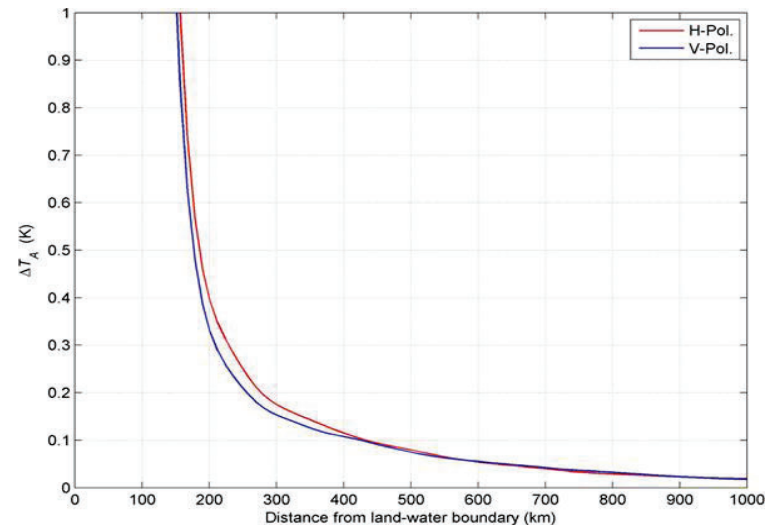

Figure 2: The effect of land contamination on the observed brightness temperature. 
The goal here is to retrieve salinity closer to shore without being critically dependent on an accurate model for land emission to correct for land contribution in the side lobes.

\section{BRIGHTNESS TEMP RECONSTRUCTION}

The alternative approach investigated here is to take advantage of the oversampling in the along-track dimension inherent in the Aquarius data collection. This is done by converting (1) to a discrete integral equation and attempting to invert it. The integral equation is ill-posed but can be inverted by regularization. The advantage of this approach is that it does not require an accurate model for emission from land in the FOV.

The first step is to rewrite (1) in terms of the reference grid shown in Fig 3. The box is chosen to be about 2.5 times the 3 $\mathrm{dB}$ width and several beam width long. In order to simplify the mapping, the brightness temperature is expanded in a Taylor series with respect to incidence angle about boresight. Keeping first order terms, one obtains:

$$
\begin{aligned}
\boldsymbol{T}_{A}\left(\boldsymbol{r}_{i}\right) & \approx \int_{G} \mathbf{G}_{P}\left(\boldsymbol{x}-\boldsymbol{r}_{i}, \boldsymbol{b}_{o}\right) \boldsymbol{T}_{B}\left(\boldsymbol{x}, \boldsymbol{b}_{o}\right) d \boldsymbol{x} \\
& +\int_{G} \mathbf{G}_{P}\left(\boldsymbol{x}-\boldsymbol{r}_{i}, \boldsymbol{b}_{o}\right)\left[c_{1} \vartheta+c_{2} \vartheta^{2}\right] d \boldsymbol{x}
\end{aligned}
$$

where $\vartheta$ is the difference between the incidence angle at point $\boldsymbol{x}$ and the incidence angle, $\vartheta_{o}$ at boresight point $\boldsymbol{r}_{i}$, and the $c_{\mathrm{i}}$ are the derivatives of the brightness temperature evaluated at boresight. Since the angular dependence of the brightness temperature is only weakly dependent on the value of $\boldsymbol{T}_{\boldsymbol{B}}$ itself, and approximate value can be used to compute these coefficients.

Assuming the antenna pattern, $\mathbf{G}_{P}$, and the $c$ 's are known, the second term is known and the integral equation (1) can be transformed to the convolution below:

$$
\boldsymbol{T}_{A o}\left(\boldsymbol{r}_{i}\right) \approx \int_{G} \mathbf{G}_{P}\left(\boldsymbol{x}-\boldsymbol{r}_{i}, \boldsymbol{b}_{o}\right) \boldsymbol{T}_{B o}(\boldsymbol{x}) d \boldsymbol{x}
$$

where $\boldsymbol{T}_{A o}$ is $\boldsymbol{T}_{A}$ minus the second integral in the previous expression and $\boldsymbol{T}_{B o}=\boldsymbol{T}_{B}\left(\boldsymbol{x}, \boldsymbol{b}_{o}\right)$ is the unknown brightness temperature field at the fixed incidence angle $\vartheta_{o}$. Solutions to (3) are sought using a uniform step quadrature to transform (3) to the linear algebraic system:

$$
\widetilde{\boldsymbol{T}}_{A o}=\boldsymbol{K} \widetilde{\boldsymbol{T}}_{B o}
$$

where $\boldsymbol{K}$ is the matrix corresponding to the quadrature in (3) and $\widetilde{\boldsymbol{T}}_{B o}$ is the column vector of unknown brightness temperatures at the discrete quadrature grid points of the region $G$ shown in Fig.3.

There are $N_{x}$ grid points along track and $N_{y}$ grid points cross track. Then $\widetilde{\boldsymbol{T}}_{B o}$ is a vector of $2 N_{x} N_{y}$ elements (the factor of 2 is for the two polarizations). Assuming there are $N_{m}$ measurements of the antenna temperature for each polarization, then $\widetilde{\boldsymbol{T}}_{A o}$ is a vector of $2 N_{m}$ elements. Since $N_{m}<N_{x} N_{y}$, the linear system in (3) is rank deficient.

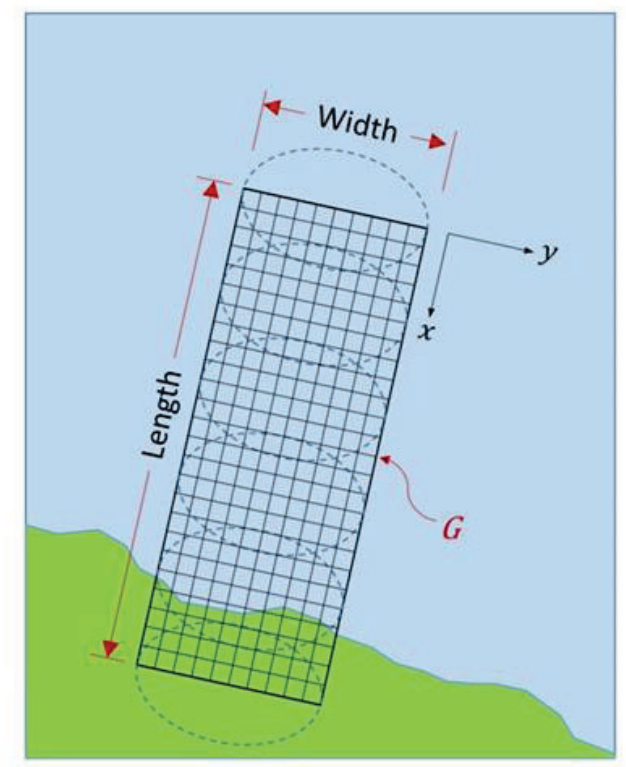

Figure 3: Study region with discrete grid

The brightness temperature $\widetilde{\boldsymbol{T}}_{B o}$ is reconstructed with the help of assumptions which provide the additional equations required to regularize the system in (3). The following assumptions are made about the solution:

- Smoothness along track,

- Flatness cross track (no cross track information)

- The polarizations are dependent (polarization consistency)

- $\quad$ Proximity to some initial estimate $\widetilde{\boldsymbol{T}}_{B \boldsymbol{E}}$

With these assumptions the regularized solution to the system in (4) is given by:

$$
\widetilde{\boldsymbol{T}}_{B o}=\boldsymbol{K}^{\dagger} \widetilde{\boldsymbol{T}}_{A o}+\boldsymbol{L}^{-1} \boldsymbol{E}^{T} \boldsymbol{E} \widetilde{\boldsymbol{T}}_{B E}
$$

where

$$
\boldsymbol{K}^{\dagger}=\boldsymbol{L}^{-1} \boldsymbol{K}^{T}
$$

and

$$
\begin{aligned}
\boldsymbol{L} & =\boldsymbol{K}^{T} \boldsymbol{K}+\boldsymbol{E}^{T} \boldsymbol{E}+\boldsymbol{C}^{T} \boldsymbol{C} \\
& +\alpha_{x} \boldsymbol{D}_{2, x}^{T} \boldsymbol{D}_{2, x}+\alpha_{y} \boldsymbol{D}_{1, y}^{T} \boldsymbol{D}_{1, y}
\end{aligned}
$$

Here, $\boldsymbol{C}$ is a diagonal matrix with entries $\alpha_{c}$ over water to provide polarization consistency, $\boldsymbol{D}_{2, x}$ is a $2^{\text {nd }}$ order finite difference matrix for smoothness along the $x$-direction (along track), $\boldsymbol{D}_{1, y}$ is a $1^{\text {st }}$ order finite difference matrix for flatness along the $y$-direction (across track) and $\boldsymbol{E}$ is a diagonal matrix with entries $\alpha_{l}$ and $\alpha_{w}$ over land and water, respectively, for proximity to the estimate $\widetilde{\boldsymbol{T}}_{B \boldsymbol{E}}$. 
The reconstructed solution in (4), requires five regularization parameters. These are namely, $\alpha_{c}, \alpha_{l}, \alpha_{w}, \alpha_{x}$ and $\alpha_{y}$. In this study, they are determined manually by trial and error.

\section{RECONSTRUCTION EXAMPLE}

Example cases have been simulated to demonstrate the feasibility of the proposed method. In the examples the grid width is $\pm 10^{\circ}$ which contains slightly more than $98 \%$ of total power in the Aquarius antenna beam; and the grid length is about $1131 \mathrm{~km}$ corresponding to 101 of the $1.44-\mathrm{sec}$ data points $\left(N_{m}=101\right)$. The grid is divided into $N_{x}=101$ by $N_{y}=11$ points for a total of 1111 grid points. The Aquarius outer beam is used in the examples.

The "data" are simulated antenna temperatures using the actual Aquarius antenna patterns and models for emission from the surface. This permits a way to test the approach because the surface brightness temperature is known. The ocean surface model uses sea surface temperature from NCEP [5] and salinity from HYCOM [6]. The land surface model uses NCEP values for temperature and soil moisture with a vegetation canopy using parameters from ECOCLIMAP [7].

Finally, the estimate $\widetilde{\boldsymbol{T}}_{B E}$ is a constant determined from these emission models by adding bias ( 0.5 psu for salinity, $5 \%$ for soil moisture and $5 \mathrm{~K}$ for land surface temperature) and random error to the ancillary data and taking the mean of the resulting brightness temperatures for land and sea.

In the example below, we consider a land crossing in the vicinity of the Amazon plume which is a dynamic area where large amounts of fresh water pour into the sea and significant salinity variations occur over relatively short distances. The simulated scene is shown in Fig 4 (left) for V-pol. It was generated using ancillary data for August 27, 2011. The Amazon River delta is just below the southern edge of the map. The dashed line is the beam center (boresight) for the Aquarius outer beam.

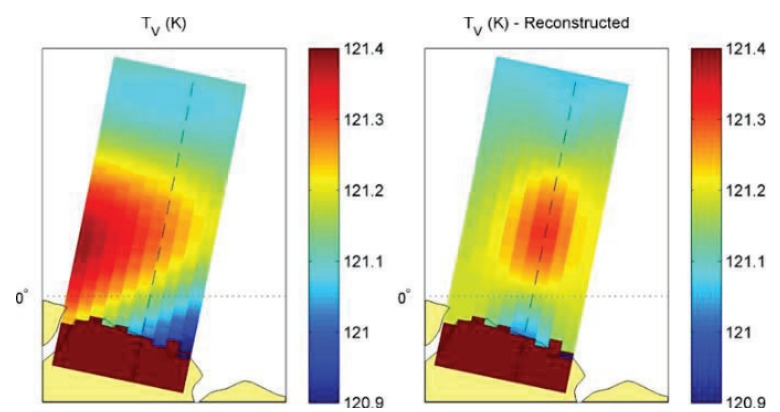

Figure 4: Original scene (left) and reconstructed image (right). The simulation is for V-pol and the Aquarius outer beam.

Fig 4 (right) shows the reconstructed image for V-pol. The reconstruction along the boresight reproduces the scene quite well, although, the quality of the reconstruction degrades as one moves to the side away from boresight. This is to be expected because the image is two dimensional but Aquarius only provides data in one dimension (along track in time). The reconstruction has been forced to be uniform across track to address this problem (the assumption of flatness across track listed above).

However, along track the reconstruction near boresight is very good. This can be seen more clearly in Figure 5 which shows the scene brightness temperature along boresight (" $x$ ") and the reconstructed brightness temperature (red) as a function of the distance from the land-water boundary. The error is much less than $0.1 \mathrm{~K}$ right up to the land-ocean boundary. This was done starting with an initial constant bias in the estimate for land of $5 \%$ for soil moisture and $5 \mathrm{~K}$ for surface temperature. The blue curves indicate the effect of adding $0.2 \mathrm{~K}$ of noise to the measurements of antenna temperature. The 95\% confidence interval (blue curves) are within $0.1 \mathrm{~K}$ right up to the land-ocean boundary.

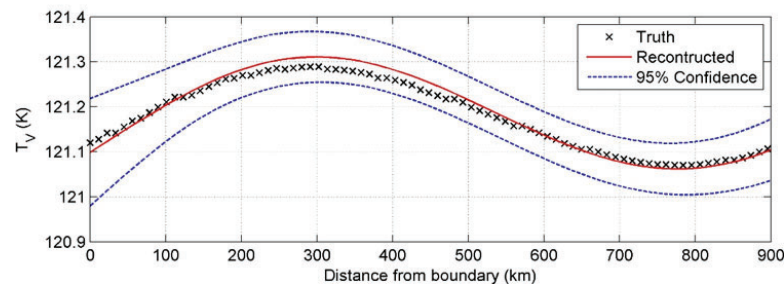

Figure 5: Performance of the reconstructed brightness temperature along boresight as a function of distance from the land-water boundary.

\section{CONCLUSIONS}

The test with simulated data suggests the approach for retrieving salinity closer to shore is promising. The approach provides enhanced spatial resolution, but the goal is to retrieve salinity closer to shore. The next step is to test the procedure with actual Aquarius data.

\section{REFERENCES}

[1] D.M. Le Vine, G.S.E. Lagerloef, R. Colomb, S. Yueh, F. Pellerano, "Aquarius: An instrument to monitor sea surface salinity from space", IEEE Trans. Geosci. Remote Sens., vol 45 (\#7), pp. 2040-2050, July, 2007.

[2] G.S.E. Lagerloef et al, "The Aquarius/SAC-D mission: Designed to meet the salinity remote sensing challenge", Oceanography, Vol21 (\#1), pp 69-81, March, 2008.

[3] D.M. Le Vine, E.P. Dinnat, S. Abraham, P. de Matthaeis and F.J. Wentz, "The Aquarius simulator and cold-sky calibration", IEEE Trans. Geosci. Remote Sens., Vol 49 (\#9), pp. 3198-3210, September, 2011.

[4] F.T. Ulaby, R.K. Moore and A.K Fung, Microwave Remote Sensing, Addison-Wesley, Vol 1, Section 6.7, 1981.

[5] National Centers for Environmental Predictxion (NCEP): www.nco.ncep.noaa.gov/pmb/products/

[6] E. P. Chassignet et al., "The HYCOM (HYbrid Coordinate Ocean Model) data assimilative system," Journal of Marine Systems, vol. 65, Issues 1-4, pp. 60-83, March 2007; also: hycom.org/dataserver

[7] Masson, V., J.L. Champeaux, F. Chauvin, C. Meriguet and R. Lacaze, 2003: A global database of land surface parameters at $1-\mathrm{km}$ resolution in mereorological and climate models, J. Clim., 16, 1261-1282. 